\title{
Comparative mycobacterial genomics as a tool for drug target and antigen discovery
}

\author{
S.T. Cole
}

Comparative mycobacterial genomics as a tool for drug target and antigen discovery. S.T. Cole. (C) ERS Journals Ltd 2002.

ABSTRACT: Genomics and the associated downstream technologies are generating vast data sets that provide new opportunities for understanding and combating both infectious and genetic diseases in humans.

The genomic approach has been applied to tuberculosis, a major cause of transmissible morbidity and mortality, with notable success. Complete genome sequences are now available for three members of the Mycobacterium tuberculosis complex and the related intracellular pathogen $M$. leprae.

Many of the predictions generated in silico by genomics have been validated through functional analysis, including studies of the transcriptome and proteome, and led to the identification of essential genes. Knowledge of the latter defines potential targets for new and existing drugs and their specificity can be assessed by comparative genomics with the host or other pathogens. Genomics is also furthering tuberculosis vaccine development by pinpointing potentially antigenic proteins as well as providing better diagnostic tools to detect infection.

Eur Respir J 2002; 20: Suppl. 36, 78s-86s.
Correspondence: S.T. Cole, Unité de Génétique Moléculaire Bactérienne, Institut Pasteur, 28 rue du Docteur Roux, 75724 Paris Cedex 15, France. Fax: 33140613583

E-mail: stcole@pasteur.fr

Keywords: Drug targets functional genomics

genomics

leprosy

tuberculosis

Received: January 282002

Accepted after revision: March 132002
There is an ever-growing need for new drugs and vaccines to treat and prevent mycobacterial diseases, particularly tuberculosis (TB), and for improved diagnostic tools to detect infection more reliably. The 1990s saw the widespread emergence of multidrug-resistant strains of Mycobacterium tuberculosis (MDR-TB) in both the developing and industrialised nations [1, 2]. Transmission of MDR-TB has been documented, particularly among the human immunodeficiency virus (HIV)-infected, and the spectre of untreatable disease is fast becoming a reality. Although it is widely accepted that vaccination is the most desirable means of preventing TB, there is extensive evidence indicating that the current vaccine, bacille Calmette-Guérin (BCG), is only effective against the rarer, disseminated forms of the disease $[3,4]$. BCG has very limited efficacy against pulmonary TB that accounts for most of the disease burden [5] and has consistently failed to confer significant protection in developing countries despite inducing protective responses against leprosy in the same settings [6]. The latter observation indicates that the leprosy bacillus probably shares common antigens with BCG and, in turn, with its close relative $M$. tuberculosis.

\section{Tuberculosis drug development: ancient and modern}

Most of the antituberculous drugs in current use were developed in the 1950s and arose from microbiological screens of libraries of natural or chemical compounds, or as the result of serendipitous leads. In 1943, streptomycin, the first antibiotic discovered, was shown to cure infections due to some Gram negative bacteria, and shortly after to be highly potent against $M$. tuberculosis. The chance observation [7] that nicotinamide inhibited the growth of mycobacteria in mice led to the synthesis and testing of related compounds, and culminated in the exquisitely specific drug isoniazid (INH) [8]. Activity testing was initially performed on in vitro grown organisms and then confirmed in an animal model of infection before clinical trials were undertaken with promising molecules. Subsequently, additional drugs were developed that inhibited particular features of $M$. tuberculosis, such as the biogenesis of its remarkable cell wall. Ideally, antibacterial agents display bactericidal activity and target essential activities. One means of pinpointing such functions, which has never been applied to the tubercle bacillus, is to isolate and characterise mutants with conditionally lethal defects and then to screen for inhibitors capable of generating the same effect. This could be done by monitoring microbiological parameters, such as growth rate, or by using an in vitro assay if suitable functional tests exist. Nowadays, identification of new drugs can result from a rational, hypothesis-driven approach inspired by genomics or from high-throughput screening of chemical or combinatorial libraries by a variety of automated methods. The desired properties of new antitubercular agents include reduction of the duration of treatment, as well as activity against latent TB infections and MDR-TB strains [9]. 


\section{Genomics}

Genomics, the systematic study of the complete set of genetic material in the cell, through deoxyribonucleic acid (DNA) sequencing and bioinformatic analysis, offers vast potential in terms of drug target and antigen discovery and is enhancing the development of new antibacterial agents and vaccines. For a relatively modest, single investment the entire complement of genes present within a pathogen can be defined and their sequences compared with those in the genome sequences of other organisms including microbes, mice and men $[10,11]$. In the field of TB research, genomics was first applied to H37Rv [12], the widely used paradigm strain of $M$. tuberculosis, and then later to CDC1551 [13], a recent clinical isolate from the USA, and then to the AF2122/97 strain of $M$. bovis [14], which was responsible for TB epidemics among cattle and badgers in the UK (table 1). The genome sequence of a close relative of the tubercle bacillus, $M$. leprae, has also been determined [15].

\section{Genomics and drug target discovery}

The genomes of all three fully-sequenced members of the $M$. tuberculosis complex contain $\sim 4.4(+/-0.1)$ $\mathrm{Mb}$ and harbour $\sim 4,000$ genes that are predicted to encode proteins and 50 genes for stable ribonucleic acid (RNA) species. In the case of M. tuberculosis $\mathrm{H} 37 \mathrm{Rv}$, bioinformatic analysis resulted in the attribution of precise functions to $\sim 40 \%$ of the 4,000 genes. Some functional information was inferred for a further $20 \%$, but nothing was learned about the remaining $40 \%[12,16]$. When functional information was available, it often enabled investigators to identify potential drug targets on the basis of their proposed biological role or their similarity to known bacterial drug targets. However, now that more mycobacterial sequences are becoming available, it is possible to establish which genes are generally found in mycobacteria or restricted to a given species $[17,18]$. The functions encoded by these genes, if essential, could represent novel targets for chemotherapy that are exceptionally specific. In contrast, a large number of genes of unknown function have been found in many bacteria and these are commonly known as the conserved hypothetical genes $[19,20]$. Their widespread conservation is certainly of biological significance and some of these genes were subsequently found to play critical roles. Thus, they represent novel targets for new broad spectrum antibiotics [19]. To ensure that new antibiotics inhibit functions that are confined to bacteria, thereby reducing potential side-effects in humans, it is now possible to perform in silico screening of the human genome sequence $[10,11]$ to exclude the possibility that related genes or proteins will be found in the host. Similar screens of the genome sequences of other pathogens can be undertaken to enhance specificity. Among the many attractive features of highly specific drugs, like INH or pyrazinamide, are the avoidance of transferable drug resistance mechanisms, such as those that have plagued certain broad spectrum antibiotics, and the reduction of unwanted side-effects, like the indiscriminate destruction of the bowel flora.

\section{Validating drug targets through functional genomics}

Several different approaches are available to determine which genes of $M$. tuberculosis are essential and thus worthy of further investigation as targets for drug development. These include gene knockouts, transcript analysis and definition of the proteome. Following is a brief description of the strategies available. Having identified potential candidates, it is important to demonstrate that the genes are expressed, particularly during infection, and here transcriptomics and proteomics offer great promise by allowing global analyses to be undertaken. All of these approaches are considerably facilitated by the availability of the complete genome sequence [12].

Transcriptomics. Various methods have been used to monitor the transcriptome, the complete set of RNA molecules produced by tubercle bacilli, including both targeted and random approaches. Among the latter are differential expression of customised amplification libraries (DECAL) [21] and selective capture of transcribed sequences (SCOTS) [22], techniques that combine polymerase chain reaction (PCR) and subtractive hybridisation in order to identify genes that are expressed differentially. When DECAL was applied to detect differences in expression in response to treatment with $\mathrm{INH}$, three overexpressed genes (ini $A$, iniB and $i n i C$ ) whose expression was also induced by ethambutol, another drug targeting the cell envelope, and by other treatments that influence cell

Table 1.-Websites with information pertinent to genomics transcriptomics, proteomics and structrual genomics of Mycobacterium tuberculosis

\begin{tabular}{|c|c|}
\hline Genomics & $\begin{array}{c}\text { http://genolist.pasteur.fr/TubercuList/ } \\
\text { http://www.sanger.ac.uk/Projects/M_tuberculosis/ } \\
\text { http://www.tigr.org/tigr-scripts/CMR2/GenomePage3.spl?database=gmt } \\
\text { http://www.sanger.ac.uk/Projects/M_bovis/ } \\
\text { http://genolist.pasteur.fr/Leproma/ } \\
\text { http://www.sanger.ac.uk/Projects/M_leprae/ }\end{array}$ \\
\hline Transcriptome & None available yet \\
\hline Structural genomics & $\begin{array}{c}\text { http://www.doe-mbi.ucla.edu/TB/ } \\
\text { http://www.pasteur.fr/X-TB/ }\end{array}$ \\
\hline Proteome & $\begin{array}{l}\text { http://www.mpiib-berlin.mpg.de/2D-PAGE/ } \\
\text { http://www.ssi.dk/en/forskning/tbimmun/tbhjemme.htm }\end{array}$ \\
\hline
\end{tabular}


wall functions, were found [21]. This suggested that the role of iniABC may be pivotal in cell envelope biogenesis, although its function remains unknown.

On application of SCOTS to detect genes differentially expressed in human primary macrophages [22], it was established that several genes were upregulated. Among these were two alternative sigma factors ( $\operatorname{sig} E$ and $\operatorname{sig} H$ ) that had been previously implicated in stress survival [23], a polyketide synthase ( $p k s 2)$, isocitrate lyase (ace $A$ also known as $i c l$ ), an enzyme that has been shown to be required for long-term persistence of $M$. tuberculosis in infected mice [24], and $m c e l B$, a gene locus that has been shown to confer the ability to invade and survive within HeLa cells to Escherichia coli [25]. Of these candidates, isocitrate lyase, whose crystal structure is available [26], appears to be the most promising drug target as it intervenes in the glyoxylate shunt, a biochemical pathway confined to certain microbes and plants.

DNA microarrays are a powerful tool for studying differential gene expression and typically consist of gene-specific probes, immobilised on a solid surface such as glass, which serve as hybridisation templates [27]. Microarrays can be used to compare gene expression in vivo with that observed in vitro, although results from $M$. tuberculosis growing in tissue have not yet been reported. However, in a most elegant study, Wilson et al. [28] examined the transcriptional response of the tubercle bacillus to INH. Overexpression of genes encoding components of the FAS-II fatty acid synthase system was detected, as expected from findings of proteomics [29], together with $f b p C$, which encodes the abundantly secreted antigen 85-C that has trehalose-dimycolyl transferase activity and intervenes in the final steps of mycobacterial cell wall synthesis [30]. Other INH-induced genes were fadE23 and fadE24, encoding two acyl-coenzyme A dehydrogenases involved in the $\beta$-oxidation of fatty acids, and $a h p C$, encoding alkyl-hydroperoxide reductase, which may respond to a secondary toxic effect of INH. Two other sets of upregulated genes deserve further comment: efp $A$, encoding a putative efflux system that may play a role in innate drug resistance [28] and ini $A B$, which were identified by DECAL [21] and are discussed above. As can be seen from these studies, microarrays and related transcriptome tools are extremely useful for identifying coregulated genes and could help pinpoint sets of genes and proteins that act cooperatively. This represents a powerful means of uncovering additional drug targets within the same pathway, thereby enhancing the chances of synergistic effects.

\section{Proteomics}

Another means of monitoring gene expression is by studying the proteome, the complete set of proteins produced by the tubercle bacilli under different conditions. The most thorough study of the proteome of different strains of the $M$. tuberculosis complex was reported by JUNGBLUT et al. [31], who used a combination of two-dimensional electrophoresis and mass spectrometry to resolve and identify proteins, respectively. Similar approaches have been applied to culture filtrate [32] and soluble proteins by ANDERSEN and coworkers [33, 34]. Details of websites providing this information are given in table 1 .

Comparative proteomics was undertaken with two different M. tuberculosis strains (H37Rv and Erdman) and two strains of M. bovis BCG (Copenhagen and Chicago). One-thousand eight-hundred spots corresponding to mycobacterial cell proteins and $>200$ inculture supernatants were detected, of which 263 and 54 were identified, respectively [31, 35]. Of the identified proteins, some perform housekeeping functions while others participate in the metabolism of fatty acids and glycolipids. Heat shock proteins were particularly abundant, whereas only three spots correspond to putative cell envelope proteins.

Proteomics can also be used as a tool for antigen discovery. Comparisons between BCG Chicago and H37Rv revealed 31 variable proteins. Thirteen were present in $\mathrm{H} 37 \mathrm{Rv}$, but not in the vaccine strain, six of which were identified. Eight proteins appeared to be absent from H37Rv, nine were downregulated, and one was overexpressed with respect to BCG. Eight of the differences were due to mobility variants, possibly caused by amino acid changes or post-translational modifications. Further comparisons between two $M$. tuberculosis strains revealed only minor differences in protein expression profiles in vitro [31]. This finding has been confirmed in an independent study in which the proteomes of the strains H37Rv and CDC1551 were compared [36].

More recently, two-dimensional liquid-phase electrophoresis has been employed to obtain several hundred fractions of culture filtrate and cytosolic proteins, respectively. These were screened for their ability to stimulate $\mathrm{T}$-cell responses and 30 individual proteins were identified by mass spectrometry, 17 of which were novel T-cell antigens [37]. At present there is little information available about the proteomes of tubercle bacilli isolated from macrophages, or even diseased tissue, and this should be investigated intensively in the coming years. Similarly, little is known about proteins present in the particulate fraction of the cell and, when one considers that $60 \%$ of known drug targets are membrane proteins, it is clear that this is an issue that needs to be addressed.

Alternative proteomic approaches exist for identifying proteins that interact or function cooperatively. Examples are the yeast two-hybrid system [38] or the tandem affinity purification (TAP) system [39]. However, to the present author's knowledge, these have not yet been applied to the tubercle bacilli on any significant scale. Protein/protein interactions can also be predicted in silico using recently developed algorithms, such as those for phylogenetic profiling, in which the distribution of given proteins in target species is examined, or by comparing target bacterial sequences with those of multidomain eukaryotic proteins to detect conserved segments that might indicate intervention in a common pathway [40-42]. A series of interesting predictions made by these techniques has been reported [43] and is now being validated experimentally. 
Gene replacement. Genes can be efficiently inactivated in tubercle bacilli by means of allelic exchange, using haploid or partially diploid hosts, mediated by suicide vectors carrying the defective allele [44] or by conditionally replicating mycobacteriophages [45]. Random gene inactivation can be achieved through conventional or "signature-tagged" transposon mutagenesis [45-47]. Sequencing the sites of insertion of large numbers of natural (IS6110) or artificial (IS1096-based) transposons provides information about nonessential genes. The signature-tagged mutagenesis method allows disruption and identification of nonessential genes that are not required in vitro but play vital roles in murine $\mathrm{TB}$ models [46, 47]. Among several thousand signature-tagged mutants tested in two independent screens, the most attenuated strains harboured transposons in genes involved in lipid metabolism and the synthesis and export of phenolphthiocerol dimycocerosate, a characteristic wax confined to pathogenic mycobacteria. Elimination of the dispensable genes leaves a set of $>2,000$ genes that are probably essential. However, given the relatively large size of the genome of $M$. tuberculosis and, above all, its long generation time, it is unlikely that the wholesale inactivation of these genes by allelic exchange will be undertaken. Another means of assessing essentiality involves the use of antisense RNA, but this has not yet seen widespread application in $M$. tuberculosis. An alternative way of identifying essential genes indirectly is to use comparative mycobacterial genomics.

\section{Comparative genomics of the Mycobacterium tuberculosis complex}

Comparative genomics is a powerful new tool for exploring microbial evolution and identifying genes that might encode new drug targets or protective antigens. Genomic diversity of the M. tuberculosis complex has been studied by DNA array technology, facilitated by the fact that all members share a $>99.95 \%$ identity at the DNA level [48]. On examination of different BCG strains, a combined total of 18 deletion regions (RD1-RD18) were uncovered by several laboratories [49-51], some of which were specific for given strains. This has led to revision of the genealogy of BCG and to the finding that 120 genes are present in $M$. tuberculosis H37Rv but absent from BCG Pasteur. This discrepancy may account for the phenotypic differences between the vaccine and the pathogen. Only one region, RD1, is missing from all BCG strains, yet present in the other $M$. tuberculosis complex members [49, 50, 52]. It is possible that loss of RD1 may account for the attenuation of $M$. bovis originally described by CAlmette [53].

Comparative genomics has also uncovered two tandem duplications of 29 and $36 \mathrm{~kb}$ (DU1, DU2) in the chromosome of $M$. bovis BCG Pasteur, indicating that this vaccine strain is partially diploid for 58 genes [54]. The combined findings of these comparative genomic analyses indicate that there is substantial genetic diversity between the various BCG strains in use today, which might account for the variability observed in different vaccine trials against TB.

Comparative genomics of the respective members of the $M$. tuberculosis complex has revealed the existence of a gene gradient. The human tubercle bacillus, $M$. tuberculosis, has more genes than $M$. africanum, $M$. microti and $M$. bovis, as these species have lost genetic material through deletion events [50, 55]. Gene loss occurs at a high frequency within the species $M$. tuberculosis as the result of homologous recombination events between copies of IS6110 that flank genes in the direct orientation $[50,55,56]$. Microarray and Affymetrix chip studies have uncovered an additional group of 45 genes whose presence, and possibly function, is facultative [51, 57]. From the combined findings it can be concluded that $>200$ genes exist that are not essential for growth of $M$. tuberculosis complex members in the host but may influence the degree of virulence.

\section{Comparative mycobacterial genomics}

An even more powerful means of reducing the number of potential new targets within $M$. tuberculosis to a more tangible level may be found by comparative genomics with the leprosy bacillus, an exceptionally slow-growing obligate intracellular pathogen that displays essentially the same cellular tropism and host range as the tubercle bacillus. At $3.27 \mathrm{Mb}$, the genome of $M$. leprae is substantially smaller than that of $M$. tuberculosis and a mere $49.5 \%$ is occupied by the 1,605 protein-coding genes [15]. $M$. leprae appears to have undergone reductive evolution, a process involving extensive downsizing and gene decay. About $27 \%$ of the M. leprae genome contains pseudogenes, inactive reading frames that still have functional counterparts in the tubercle bacillus, of which 1,114 are recognisable, while the remaining $23.5 \%$ of the genome appears to be noncoding and is probably nonfunctional. Reductive evolution has been documented in obligate intracellular pathogens and endosymbionts, such as Rickettsia and Buchnera spp., respectively [15]. Genes are progressively lost as their functions are no longer required in highly specialised niches and this probably accounts for the exceptionally long generation time of the leprosy bacillus. In summary, assuming that all mycobacteria are descended from a common ancestor, $M$. leprae has probably lost $>2,000$ genes during its evolution and the minimal gene set required by a pathogenic mycobacterium may have been defined naturally [15].

When pairwise comparisons of the gene and protein sets of the leprosy and tubercle bacilli $[12,15,16,58]$ were performed, 1,433 proteins were found to be common to both pathogens. After removal of proteins that are shared with all other prokaryotes (except Actinomycetes) and eukaryotes the sample contains only 333 proteins. Since these pathogenic mycobacteria occupy similar niches in the human body, where they encounter the same physiological stresses and immune responses, it is conceivable that the products of some of these genes may affect highly specialised functions that could be essential for 
intracellular growth of mycobacteria. If this was the case, the corresponding proteins or enzymes might represent novel drug targets. The 333 candidates identified by comparative mycobacterial genomics can be subdivided into those proteins that are confined to the genus Mycobacterium (there are 219 of these) and a second group of 114 polypeptides that also occur in Streptomyces or Corynebacteria spp., related members of the Actinomycetales kingdom. It is reasonable to assume that the latter proteins confer specific properties on actinomycetes, whereas those that are restricted to mycobacteria may play an even more specialised role.

\section{Target discovery}

To further illustrate the usefulness of comparative mycobacterial genomics for identifying potentially important proteins, two precise examples will now be given. Multiple gene duplication events have occurred in $M$. tuberculosis and when limited divergence has followed, this appears to have resulted in extensive functional redundancy in numerous biochemical pathways $[12,16,58]$. Indeed, in many cases it is difficult to predict with certitude which of two duplicated genes affects a specific function. This is true for five proteins (Rv0462, Rv0794c，Rv2855, Rv2713, Rv3303c) that show strong similarity on database searches to various lipoamide dehydrogenase components of the pyruvate dehydrogenase complex, an essential function. During the initial analysis of the $M$. tuberculosis genome, the two proteins displaying the strongest similarity to this dehydrogenase were termed $l p d A(\mathrm{Rv} 3303 \mathrm{c})$ and $l p d B$ (Rv0794c), but subsequent biochemical studies of the various gene products revealed that authentic lipoamide dehydrogenase was encoded by Rv0462 (now termed $l p d$ ) and not by the $\operatorname{lpdA}$ or $\operatorname{lpdB}$ genes [59]. Inspection of the $M$. leprae genome sequence would have helped focus this work considerably, since only one of these five $M$. tuberculosis genes has a functional orthologue, with ML2387 corresponding to Rv0462, the true lipoamide dehydrogenase. The remaining four genes are present in pseudogene form in the $M$. leprae genome $[15,60]$. This is strong testimony to the power of comparative genomics.
The following is a second example that awaits biochemical confirmation. Preproteins exported by the twin-arginine transport, or Tat, pathway generally bind redox cofactors and fold or oligomerise before crossing the membrane [61, 62]. After removal of the signal peptide, many of these proteins function in extracytoplasmic electron transfer chains. The specialised machinery that recognises the twin-arginine motif [63] and translocates the preprotein across the membrane is composed of several different Tat proteins. In E. coli, TatA and TatE are 50\% identical and share weak similarity with TatB [62]. All three proteins are predicted to be anchored to the cytoplasmic membrane via an $\mathrm{N}$-terminal hydrophobic $\alpha$-helix and to have cytoplasmic amphipathic helices followed by variable regions. The TatC protein is predicted to be an integral membrane protein with six transmembrane segments. $M$. tuberculosis and $M$. leprae both contain clearly identifiable tat $A$, tat $B$, tat $C$ and tat $D$ genes and must, therefore, produce a functional Tat system.

On examination of the proteome of $M$. tuberculosis, 11 potential substrates for the Tat export system were recognised on the basis of their signal peptides containing potential twin-arginine motifs at the N-terminus and the cognate motif, S/TRRXFLK [63] (table 2). During the extensive reductive evolution of the genome of $M$. leprae only one of the corresponding genes, ML1190, escaped inactivation. It is orthologous to Rv2525c of $M$. tuberculosis but shows no similarity to other proteins present in current sequence databases. The 240 amino acid-long precursor protein encoded by ML1190/Rv2525c contains five histidine and one cysteine residue that may be important for coordinating divalent metal ions. The conservation of this coding sequence by $M$. leprae, in the face of massive gene loss, is a strong indication that it must play an important biological role. Given the many parallels with Tat systems elsewhere, it is likely to be in electron transport. These indirect arguments suggest on the one hand that, if this function were essential, the ML1190/ Rv2525c gene product might represent a novel drug target or, on the other, since it is likely to be located extracellularly, it may be an important sentinel protein antigen.

Table 2. - Possible twin arginine secreted proteins

\begin{tabular}{lcll}
\hline M. tuberculosis & M. leprae & Gene & Predicted function \\
\hline Rv0203 & Del & & Unknown \\
Rv0265c & NF & $f e c B 2$ & Iron transport protein FeIII dicitrate transporter \\
Rv0846c & ML2171 ps & Similar to several L-ascorbate oxidases \\
Rv1755c & Del & $p l c C$ & Phospholipase C precursor \\
Rv2349c & NF & plcB & Phospholipase C precursor \\
Rv2350c & Del & plcA & Phospholipase C precursor \\
Rv2351c & NF & & Phospholipase C precursor \\
Rv2525c & ML1190 & Unknown & Similarity to G755244 acid phosphatase \\
Rv2577 & ML0497 ps & Sn-glycerol-3-phosphate transport \\
Rv2833c & Del & & Unknown \\
Rv3353c & Del & & Unknown \\
NF & ML2649 & &
\end{tabular}

NF: not found; Del: deleted; ps: pseudogene; M. tuberculosis: Mycobacterium tuberculosis; M. leprae: Mycobacterium leprae. 


\section{Screening drug targets}

If the corresponding protein has an assayable function, kinase activity for example, it can be used as the basis of an in vitro screen to identify inhibitors of the enriched or purified enzyme. The advantage of this approach is that it can generally be automated or converted to high-throughput format to facilitate screening of large or complex libraries of synthetic compounds or natural products. However, whole organism screens, involving recombinant $M$. tuberculosis strains with reporter activity, such as luciferase or green fluorescent protein [64, 65], are often considered preferable as they avoid drug permeability problems. Once an active pharmacophore has been uncovered, numerous analogues can be synthesised or identified in combinatorial libraries to isolate more active derivatives. Their potency can also be evaluated using reporter assays or biochemical techniques, such as transcriptome or proteome analysis. In this way, genes that are coregulated can be uncovered whose products may also serve as potential drug targets in turn, as they often act concertedly in the same metabolic process. Identification of the target enables large amounts of the corresponding protein to be produced by genetic engineering for further studies. Knowledge of the three-dimensional structure of known or potential drug targets is also highly desirable for drug development purposes and can be obtained through structural biology.

\section{Structural genomics}

Structural genomics is an emerging scientific discipline that exploits high-throughput cloning technology to generate systematically large numbers of expression constructs corresponding to most, or all, of the genes present in a microbial genome (table 1). These constructs are used to produce large amounts of tagged protein that can be purified in a single step by powerful affinity chromatography methods, thereby facilitating downstream structural analysis by X-ray crystallography, nuclear magnetic resonance (NMR) spectroscopy or high-resolution cryo-electron microscopy. Current experience predicts a $60 \%$ success rate at the expression stage, with $\sim 20 \%$ of purified proteins subsequently giving diffraction grade crystals. Structural genomics is a promising new approach to drug and drug-target discovery as it readily interfaces with structure-based drug design [66]. In the case of larger genomes, structural genomics generally concentrates on large protein families to maximise the yield, whereas in drugtarget discovery programmes the approach is usually hypothesis-driven.

\section{Diagnostics}

The diagnosis of both active disease, which relies heavily on clinical expertise and the detection of acidfast bacilli in sputum smears, and latent TB will also benefit from comparative genomics. Latent infection is often diagnosed by monitoring the extent of delayed type hypersensitivity reactions following intradermal injection of tuberculin, an ill-defined mixture of antigens. Tuberculin reactivity is of limited value in communities where BCG vaccination is practised and its interpretation may also be confounded by infections involving other mycobacteria. The identification of 120 genes in the tubercle bacillus [49, 50], which are absent from BCG, allows a move towards the development of a more specific test that can distinguish between infection and immunisation. Microarrays and proteomics will also find wide application in monitoring biodiversity within the $M$. tuberculosis complex and help to confirm the presence or absence of candidate diagnostic antigens.

\section{Antigen discovery and vaccine strategies}

Knowledge of the subcellular location of proteins is particularly valuable for the design of new TB vaccines, both preventive and therapeutic, since it is widely believed that surface-exposed or secreted proteins correspond to those antigenic components that are first encountered by the immune system during infection [67]. Figure 1 presents a scheme

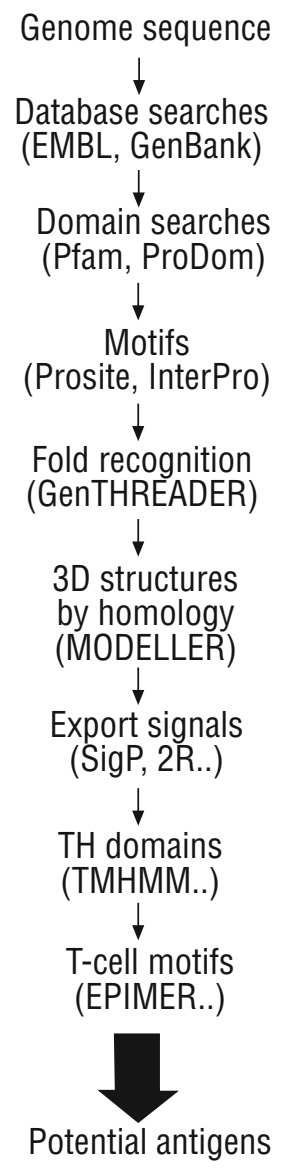

Fig. 1.-A scheme to identify potential antigenic proteins using bioinformatic tools to analyse genome data. The databases or programs used are shown in brackets. See main text for further details. 3D: three dimensional. 
whereby such proteins can be found. The system employs bioinformatics to identify proteins that localise to the cell envelope. These include transmembrane proteins with hydrophobic domains and lipoproteins with N-terminal cysteine residues that are modified by addition of lipid groups. Proteins that are secreted via the general secretory pathway [68] are readily identifiable by their characteristic signal peptides as are those metallo-enzymes that are secreted by the TAT system [61-63]. Other proteins that lack signal peptides and are secreted from mycobacteria in a Sec-independent manner include those belonging to the early secreted antigenic target (ESAT)-6 family $[12,58]$. ESAT-6 is a potent $\mathrm{T}$-cell antigen that induces strong $\mathrm{T}$-helper 1-type responses [69] and has been extensively studied as a potential diagnostic reagent for infection [70], since its gene is missing from BCG [50, 51, 53, 71], and as a component of a subunit vaccine [72]. In addition to highly purified proteins that are envisaged for use as subunit vaccine components, other strategies are being pursued. These include nucleic acid vaccines [73, 74], the construction of rationally attenuated mutants of M. tuberculosis, and improved BCG strains [75]. All of these approaches have been boosted by the availability of the genome sequence [12].

\section{Appraisal of immunogenicity in silico and in vivo}

Comparative in silico analysis of the proteome of Mycobacterium tuberculosis has reduced the number of potential subunit vaccine candidates from $\sim 4,000$ proteins to $<40$ potentially exported polypeptides, which are present in both the leprosy and tubercle bacilli. Several of these belong to protein families of conserved sequence that are invariably larger in Mycobacterium tuberculosis than in Mycobacterium leprae. The comparative genomic analysis has highlighted the potential importance of the esterase activator-6 proteins [12, 58], together with their likely secretion machinery, and initial immunological characterisation has yielded encouraging results [76]. To establish which of these protein candidates contain potential T-cell epitopes, powerful new algorithms such as EpiMer [77], can be used to further refine the selection. The availability of the genome sequences of both mice and humans $[10,11]$ will allow potentially crossreactive epitopes or antigens to be identified in silico, thereby limiting possible complications. Ultimately, the success of the approach will require testing the candidates retained in cellular and animal models for tuberculosis, and establishing the appropriate correlates of protection.

\footnotetext{
Acknowledgements. The author would like to give special thanks to the mycobacterial genomics groups at the Institut Pasteur and the Sanger Centre. Parts of this work were supported by the Wellcome Trust, the Institut Pasteur, the European Community (QLK2CT-1999-01093, QLRT-2001-02018), and the Association Française Raoul Follereau.
}

\section{References}

1. Dye C, Sheele S, Dolin P, Pathania V, Raviglione MC. Global burden of tuberculosis: estimated incidence, prevalence, and mortality by country. JAMA 1999; 282: 677-686.

2. Espinal MA, Laszlo A, Simonsen L, et al. Global trends in resistance to antituberculosis drugs. $N$ Engl $J$ Med 2001; 344: 1294-1303.

3. Bloom BR, Fine PEM. The BCG experience: Implications for future vaccines against tuberculosis. In: Bloom BR, eds. Tuberculosis: Pathogenesis, protection, and control. Washington DC, American Society for Microbiology, 1994; pp. 531-557.

4. Fine PEM. Variation in protection by BCG: implications of and for heterologous immunity. Lancet 1995; 346: 1339-1345.

5. Styblo K. Impact of BCG vaccination programmes in children and young adults on the tuberculosis program. Tubercle 1976; 57: 17-43.

6. Anon. Randomised controlled trial of single BCG, repeated BCG, or combined BCG and killed Mycobacterium leprae vaccine for prevention of leprosy and tuberculosis in Malawi. Karonga Prevention Trial Group. Lancet 1996; 348: 17-24.

7. Chorine V. Action of nicotinamide on bacilli of the genus Mycobacterium. Compt Rendu Acad Sci 1945; 220: $150-152$.

8. Fox HH. Synthetic tuberculostatics show promise. Chem Eng News 1951; 29: 3963-3964.

9. Global Alliance for TB Drug Development. Scientific blueprint for TB drug development. Tuberculosis 2001; 81: Suppl. 1, 1-52.

10. International Human Genome Sequencing Consortium. Initial sequencing and analysis of the human genome. Nature 2001; 409: 860-921.

11. Venter CJ, Adams MD, Myers EW, et al. The sequence of the human genome. Science 2001; 291: 1304-1351.

12. Cole ST, Brosch R, Parkhill J, et al. Deciphering the biology of Mycobacterium tuberculosis from the complete genome sequence. Nature 1998; 393: 537544.

13. Fleischmann RD, Alland D, Eisen JA, et al. Whole genome comparison of Mycobacterium tuberculosis clinical and laboratory strains. J Bacteriol 2002 (in press).

14. Gordon SV, Eiglmeier K, Garnier T, et al. Genomics of Mycobacterium bovis. Tubercle Lung Dis 2001; 6: 157-163.

15. Cole ST, Eiglmeier K, Parkhill J, et al. Massive gene decay in the leprosy bacillus. Nature 2001; 409: 10071011.

16. Cole ST. Learning from the genome sequence of Mycobacterium tuberculosis H37Rv. FEBS Letters 1999; 452: 7-10.

17. Cole ST. Comparative mycobacterial genomics. Curr Opin Microbiol 1998; 1: 567-571.

18. Brosch R, Pym AS, Gordon SV, Cole ST. The evolution of mycobacterial pathogenicity: clues from comparative genomics. Trends Microbiol 2001; 9: 452458.

19. Arigoni F, Talabot F, Peitsch $\mathrm{M}$, et al. A genome based approach for the identification of essential bacterial genes. Nat Biotechnol 1998; 16: 851-856.

20. Mushegian AR, Koonin EV. A minimal gene set for cellular life derived by comparison of complete 
bacterial genomes. Proc Natl Acad Sci USA 1996; 93: 10268-10273.

21. Alland D, Kramnik I, Weisbrod TR, et al. Identification of differentially expressed mRNA in prokaryotic organisms by customized amplification libraries (DECAL): the effect of isoniazid on gene expression in Mycobacterium tuberculosis. Proc Natl Acad Sci USA 1998; 95: 13227-13232.

22. Graham JE, Clark-Curtiss JE. Identification of Mycobacterium tuberculosis RNAs synthesized in response to phagocytosis by human macrophages by selective capture of transcribed sequences (SCOTS). Proc Natl Acad Sci USA 1999; 96: 11554-11559.

23. Fernandes ND, Wu QL, Kong D, Puyang X, Garg S, Husson RN. A mycobacterial extracytoplasmic sigma factor involved in survival following heat shock and oxidative stress. J Bacteriol 1999; 181: 4266-4274.

24. McKinney JD, Höner zu Bentrup K, Munoz-Elias EJ, et al. Persistence of Mycobacterium tuberculosis in macrophages and mice requires the glyoxylate shunt enzyme isocitrate lyase. Nature 2000; 406: 735-738.

25. Arruda S, Bomfim G, Knights R, Huima-Byron T, Riley LW. Cloning of an M. tuberculosis DNA fragment associated with entry and survival inside cells. Science 1993; 261: 1454-1457.

26. Sharma V, Sharma S, Hoener zu Bentrup K, et al. Structure of isocitrate lyase, a persistence factor of Mycobacterium tuberculosis. Nat Struct Biol 2000; 7 : 663-668.

27. de Risi JL, Iyer VR, Brown PO. Exploring the metabolic and genetic control of gene expression on a genomic scale. Science 1997; 278: 680-686.

28. Wilson M, de Risi J, Kristensen H-K, et al. Exploring drug-induced alterations in gene expression in Mycobacterium tuberculosis by microarray hybridization. Proc Natl Acad Sci USA 1999; 96: 6854-6859.

29. Mdluli K, Slayden RA, Zhu Y-Q, et al. Inhibition of a Mycobacterium tuberculosis $\beta$-ketoacyl ACP synthase by isoniazid. Science 1998; 280: 1607-1610.

30. Belisle JT, Vissa VD, Sievert T, Takayama K, Brennan PJ, Besra GS. Role of the major antigen of Mycobacterium tuberculosis in cell wall biogenesis. Science 1997; 276: 1420-1422.

31. Jungblut PR, Schaible UE, Mollenkopf H-J, et al. Comparative proteome analysis of Mycobacterium tuberculosis and Mycobacterium bovis BCG strains: towards functional genomics of microbial pathogens. Mol Microbiol 1999; 33: 1103-1117.

32. Weldingh K, Rosenkrands I, Jacobsen S, Rasmussen PB, Elhay MJ, Andersen P. Two-dimensional electrophoresis for analysis of Mycobacterium tuberculosis culture filtrate and purification and characterization of six novel proteins. Infect Immun 1998; 66: 3492-3500.

33. Rosenkrands I, King A, Weldingh K, Moniatte M, Moertz E, Andersen P. Towards the proteome of Mycobacterium tuberculosis. Electrophoresis 2000; 21: 3740-3756.

34. Rosenkrands I, Weldingh $\mathrm{K}$, Jacobsen S, et al. Mapping and identification of Mycobacterium tuberculosis proteins by two-dimensional gel electrophoresis, microsequencing and immunodetection. Electrophoresis 2000; 21: 935-948.

35. Mattow J, Jungblut PR, Muller EC, Kaufmann SH. Identification of acidic, low molecular mass proteins of Mycobacterium tuberculosis strain H37Rv by matrix-assisted laser desorption/ionization and electrospray ionization mass spectrometry. Proteomics 2001; 1: 494-507.

36. Betts JC, Dodson P, Quan S, et al. Comparison of the proteome of Mycobacterium tuberculosis strain H37Rv with clinical isolate CDC 1551. Microbiology 2000; 146: 3205-3216.

37. Covert BA, Spencer JS, Orme IM, Belisle JT. The application of proteomics in defining the $\mathrm{T}$ cell antigens of Mycobacterium tuberculosis. Proteomics 2001; 1: 574-586.

38. Evangelista C, Lockshon D, Fields S. The yeast twohybrid system: prospects for protein linkage maps. Trends Cell Biol 1996; 6: 196-199.

39. Rigaut G, Shevchenko A, Rutz B, Wilm M, Mann M, Seraphin B. A generic protein purification method for protein complex characterization and proteome exploration. Nat Biotechnol 1999; 17: 1030-1032.

40. Marcotte EM, Pellegrini M, Thompson MJ, Yeates TO, Eisenberg D. A combined algorithm for genomewide prediction of protein function. Nature 1999; 402: 83-86.

41. Marcotte EM, Xenarios I, van Der Bliek AM, Eisenberg D. Localizing proteins in the cell from their phylogenetic profiles. Proc Natl Acad Sci USA 2000; 97: 12115-12120.

42. Enright AJ, Iliopoulos I, Kyrpides NC, Ouzounis CA. Protein interaction maps for complete genomes based on gene fusion events. Nature 1999; 402: 86-90.

43. Xenarios I, Salwinski L, Duan XJ, Higney P, Kim SM, Eisenberg D. DIP, the Database of Interacting Proteins: a research tool for studying cellular networks of protein interactions. Nucleic Acids Res 2002; 30: 303-305.

44. Pelicic V, Jackson M, Reyrat JM, Jacobs WR Jr, Gicquel B, Guilhot C. Efficient allelic exchange and transposon mutagenesis in Mycobacterium tuberculosis. Proc Natl Acad Sci USA 1997; 94: 10955-10960.

45. Bardarov S, Kriakov J, Carriere C, et al. Conditionally replicating mycobacteriophages: a system for transposon delivery to Mycobacterium tuberculosis. Proc Natl Acad Sci USA 1997; 94: 10961-10966.

46. Camacho LR, Ensergueix D, Perez E, Gicquel B, Guilhot C. Identification of a virulence gene cluster of Mycobacterium tuberculosis by signature-tagged transposon mutagenesis. Mol Microbiol 1999; 34: 257-267.

47. Cox JS, Chen B, McNeil M, Jacobs WR Jr. Complex lipid determines tissue-specific replication of Mycobacterium tuberculosis in mice. Nature 1999; 402: 79-83.

48. Sreevatsan S, Pan X, Stockbauer KE, et al. Restricted structural gene polymorphism in the Mycobacterium tuberculosis complex indicates evolutionarily recent global dissemination. Proc Natl Acad Sci USA 1997; 94: 9869-9874.

49. Behr MA, Wilson MA, Gill WP, et al. Comparative genomics of BCG vaccines by whole-genome DNA microarrays. Science 1999; 284: 1520-1523.

50. Gordon SV, Brosch R, Billault A, Garnier T, Eiglmeier K, Cole ST. Identification of variable regions in the genomes of tubercle bacilli using bacterial artificial chromosome arrays. Molec Microbiol 1999; 32: 643-656.

51. Salamon H, Kato-Maeda M, Small PM, Drenkow J, Gingeras TR. Detection of deleted genomic DNA using a semiautomated computational analysis of GeneChip data. Genome Res 2000; 10: 2044-2054.

52. Mahairas GG, Sabo PJ, Hickey MJ, Singh DC, 
Stover CK. Molecular analysis of genetic differences between Mycobacterium bovis BCG and virulent M. bovis. J Bacteriol 1996; 178: 1274-1282.

53. Calmette A. Preventive vaccination against tuberculosis. Paris, Masson et cie, 1927; pp. 1-250.

54. Brosch R, Gordon SV, Buchrieser C, Pym A, Garnier T, Cole ST. Comparative genomics uncovers tandem chromosomal duplications in some strains of $\mathrm{Myco}-$ bacterium bovis BCG: Implications for vaccination. Comp Funct Genomics (Yeast) 2000; 17: 111-123.

55. Brosch R, Gordon SV, Eiglmeier K, et al. Genomics, biology, and evolution of the Mycobacterium tuberculosis complex. In: Hatfull GF, Jacobs WR Jr, eds. Washington DC, ASM Press, 2000; pp. 19-36.

56. Brosch R, Philipp W, Stavropolous E, Colston MJ, Cole ST, Gordon SV. Genomic analysis reveals variation between Mycobacterium tuberculosis H37Rv and the attenuated $M$. tuberculosis H37Ra. Infect Immun 1999; 67: 5768-5774.

57. Kato-Maeda M, Rhee JT, Gingeras TR, et al. Comparing genomes within the species Mycobacterium tuberculosis. Genome Res 2001; 11: 547-554.

58. Tekaia F, Gordon SV, Garnier T, Brosch R, Barrell BG, Cole ST. Analysis of the proteome of Mycobacterium tuberculosis in silico. Tubercle Lung Disease 1999; 79: 329-342.

59. Argyrou A, Blanchard JS. Mycobacterium tuberculosis lipoamide dehydrogenase is encoded by Rv0462 and not by the $l p d A$ or $l p d B$ genes. Biochemistry 2001; 40: 11353-11363.

60. Jones LM, Cole ST, Moszer I. Leproma: A Mycobacterium leprae genome browser. Lep Rev 2001; 72 : 470-477.

61. Berks BC, Sargent F, De Leeuw E, et al. A novel protein transport system involved in the biogenesis of bacterial electron transfer chains. Biochim Biophys Acta 2000; 1459: 325-330.

62. Berks BC, Sargent F, Palmer T. The Tat protein export pathway. Mol Microbiol 2000; 35: 260-274.

63. Stanley NR, Palmer T, Berks BC. The twin arginine consensus motif of Tat signal peptides is involved in Sec-independent protein targeting in Escherichia coli. J Biol Chem 2000; 275: 11591-11596.

64. Shawar RM, Humble DJ, Van Dalfsen JM, et al. Rapid screening of natural products for antimycobacterial activity by using luciferase-expressing strains of Mycobacterium bovis BCG and Mycobacterium intracellulare. Antimicrob Agents Chemother 1997; 41: 570-574.
65. Snewin VA, Gares MP, Gaora PO, Hasan Z, Brown IN, Young DB. Assessment of immunity to mycobacterial infection with luciferase reporter constructs. Infect Immun 1999; 66: 4586-4593.

66. Kubinyi H. Structure-based design of enzyme inhibitors and receptor ligands. Curr Opin Drug Disc Devel 1998; 1: 4-15.

67. Andersen P. Host responses and antigens involved in protective immunity to Mycobacterium tuberculosis. Scand J Immunol 1997; 45: 115-131.

68. Pugsley AP. The complete general secretory pathway in Gram-negative bacteria. Microbiol Rev 1993; 57: 50-108.

69. Lalvani A, Brookes R, Wilkinson RJ, et al. Human cytolytic and interferon gamma-secreting CD8+ T lymphocytes specific for Mycobacterium tuberculosis. Proc Nat Acad Sci USA 1998; 95: 270-275.

70. Pollock JM, Andersen P. The potential of the ESAT-6 antigen secreted by virulent mycobacteria for specific diagnosis of tuberculosis. J Inf Dis 1997; 175: 12511254.

71. Harboe M, Oettinger T, Wiker HG, Rosenkrands I, Andersen P. Evidence for occurrence of the ESAT-6 protein in Mycobacterium tuberculosis and virulent Mycobacterium bovis and for its absence in Mycobacterium bovis BCG. Infect Immun 1996; 64: 16-22.

72. Brandt L, Elhay M, Rosenkrands I, Lindblad EB, Andersen P. ESAT-6 subunit vaccination against Mycobacterium tuberculosis. Infect Immun 2000; 68: 791-795.

73. Huygen $\mathrm{K}$, Content $\mathrm{J}$, Denis $\mathrm{O}$, et al. Immunogenicity and protective efficacy of a tuberculosis DNA vaccine. Nature Medicine 1996; 2: 893-898.

74. Tascon RE, Colston MJ, Ragno S, Stavropoulos E, Gregory D, Lowrie DB. Vaccination against tuberculosis by DNA injection. Nature Medicine 1996; 2 : 888-892.

75. Baldwin SL, D'Souza C, Roberts AD, et al. Evaluation of new vaccines in the mouse and guinea pig model of tuberculosis. Infect Immun 1998; 66: 2951-2959.

76. Skjøt RLV, Oettinger T, Rosenkrands I, et al. Comparative evaluation of low-molecular-mass proteins from Mycobacterium tuberculosis identifies members of the ESAT-6 family as immunodominant T-cell antigens. Infect Immun 2000; 68: 214-220.

77. De Groot AS, Bosma A, Chinai N, et al. From genome to vaccine: in silico predictions, ex vivo verification. Vaccine 2001; 19: 4385-4395. 